\title{
Psychiatric Morbidity of Chikungunya among Children in Bangladesh
} \author{
Gias U. Ahsan ${ }^{2}$ \\ ${ }^{1}$ Institute of Child Health, Mirpur, Dhaka, Bangladesh \\ ${ }^{2}$ North South University, Dhaka, Bangladesh \\ Email: ${ }^{*}$ mohammad.hawlader@northsouth.edu
}

Sanjana Zaman 1,2, Mohammad Delwer Hossain Hawlader ${ }^{2 *}$, Ariful Bari Chowdhury'2,

How to cite this paper: Zaman, S., Hawlader, M.D.H., Chowdhury, A.B. and Ahsan, G.U. (2018) Psychiatric Morbidity of Chikungunya among Children in Bangladesh. Health, 10, 90-94.

https://doi.org/10.4236/health.2018.101007

Received: November 30, 2017

Accepted: January 15, 2018

Published: January 18, 2018

Copyright $\odot 2018$ by authors and Scientific Research Publishing Inc. This work is licensed under the Creative Commons Attribution International License (CC BY 4.0).

http://creativecommons.org/licenses/by/4.0/

(c) (i) Open Access

\begin{abstract}
Introduction: Chikungunya occurred as a major epidemic form in Bangladesh in 2017 after a gap of 6 years. The first outbreak of Chikungunya was identified in Bangladesh in 2008. Another outbreak occurred at Dhaka in 2011. But the current outbreak is huge and effecting almost two third of the population. Methods: This was a retrospective case series study, consisted of 60 children, among which 35 were male and 25 female, ages ranging from 5 to 15 years. Those children were recruited from child health care clinic with a history of two to five days' high grade fever. All patients recruited were IgM antibody for Chikungunya positive (after $5^{\text {th }}$ day of onset of fever) and on the other hand antibody for Dengue was negative. Two patients were excluded from the study due to Cerebral Palsy and Claustrophobia. Finally 58 patients were assessed. Informed written consent was obtained from all the legal guardians before data collection. Results: All patients presented with a history of two to five days' high grade fever. The fever was preceded by a maculopapular rash over the trunk and extremities, headache, myalgia and arthralgia. Delirium (non-specified) was most prevalent psychiatric symptoms 45 (77\%). Other psychiatric symptoms were Panic Disorder (56\%), Phobic Disorder (36\%), Neurasthenia or Fatigue syndrome (27\%), Hypersomnia (13\%) and Claustrophobia (5\%). Conclusion: This study suggested that psychiatric morbidity among pediatric Chikungunya patients is very common. Knowledge of the pathogenesis is required to reduce the psychiatric complications and to prevent the morbidity. There is an emergence to invent the prophylactic vaccination, proper and early diagnosis of Chikungunya.
\end{abstract}

\section{Keywords}

Chikungunya, Dengue, Psychiatric Symptoms, Children, Bangladesh 


\section{Introduction}

Chikungunya is a viral fever caused by a single stranded RNA virus of the genus alpha virus in the family Togaviridae (Arbo virus) and transmitted to human by the bite of Aedes Aegypti mosquito (yellow fever mosquito) [1]. The word "Chikungunya" is derived from the Makonde language, spoken by an ethnic group in southeast Tanzania and Northern Mozambique where the first cases of this disease were described. The word Chikungunya means "that which bends up" which refers to stooped posture, and develops as a result of arthritic symptoms of the disease. Chikungunya virus is originated from Africa [2].

The first outbreak of Chikungunya was identified in Bangladesh in 2008. In 2011, another outbreak occurred at Dhaka (Dohar Sub-district) [3]. Chikungunya occurred as a Major epidemic form in Bangladesh after a gap of 6 years in 2017. Chikungunya fever is an acute illness, mainly characterized by acute fever and severe joint pain [4] and has various complications like rheumatological, neurological, psychological, and dermatological, and even multi-organ failure. Till now psychiatric complications are not well studied. Recently, some psychiatric symptoms have been described in patients with Chikungunya fever [5]. Identified psychiatric manifestations due to Chikungunya virus infection include: insomnia, aggressiveness, pessimism, loss of concentration, depression, and mental confusion [6]. In a study carried out in India, almost $60 \%$ of the patients presented anxiety disorder or depressive episodes [5]. Neurological symptoms and signs in Chikungunya occur early in the course of disease on the 2nd or 3rd day of fever. A prospective study was performed in India from August to October 2006, and that study illustrated the occurrence of neurological symptoms and signs in cases of Chikungunya. Patients showed a disturbance in their level of consciousness, such as confusion, drowsiness and delirium [7].

\subsection{Materials and Methods}

Study type: A retrospective case series study was performed.

Sampling place: Study participants for this study were chosen from a Paediatrics clinic.

Sampling technique:

A convenience sampling technique was used. Study recruited 60 children from March 2017 to August 2017.

\subsection{Study Participants}

The children age ranged from 5 years to 15 years who came to the child health care clinic with a history of two to five days' high grade fever. The fever was preceded by a maculopapular rash over the trunk and extremities, headache, myalgia and arthralgia. All patients recruited were IgM antibody for Chikungunya positive (after the $5^{\text {th }}$ day of onset of fever) and on the other hand antibody for Dengue was negative. Two patients were excluded from the study due to Cerebral Palsy and claustrophobia. Finally 58 patients were recruited for assess- 
ment. Complete history was taken from the parents. All patients were thoroughly examined and found them presented with multiple peripheral joint arthritis (usually symmetrical). To assess the psychiatric complications, mental status examination were done by ICD-10 classification of Mental and Behavioral Disorders [World Health Organization]. The ICD-10 Classification of Mental and Behavioral Disorders: Diagnostic Criteria for Research [Geneva: WHO, 1992]. Informed written consent was taken from the parents/legal guardians. Data were categorized and analyzed using SPSS for windows version 21.0. Descriptive statistics were performed for age, sex and biochemical parameters.

\section{Ethical Consideration}

This study was approved by the ethical review committee of North South University, Dhaka, Bangladesh. Privacy and anonymity of the participants were maintained.

\section{Result}

Table 1 shows the number and percentage of the frequencies of psychiatric symptoms such as Delirium (non-specified), Panic Disorder, Phobic Disorder, Claustrophobia, Hypersomnia and Neurasthenia after Chikungunya infection. Delirium (non-specified) was most prevalent psychiatric symptoms among pediatric patients who had suffered from Chikungunya infection. 45 out of 58 patients had Delirium (77\%). Other psychiatric symptoms were Panic Disorder (56\%), Phobic Disorder (36\%), Neurasthenia or Fatigue syndrome (27\%), Hypersomnia (13\%), and Claustrophobia (5\%).

\section{Discussion}

Dermatological, rheumatologic, neurological manifestations of Chikungunya are well known but Psychiatric complications in pediatric patients with Chikungunya fever are not well known as others [8]. Our study assessed the psychiatric complications occurring during the course of Chikungunya fever. Psychiatric complications arising during the disease process provide us a conclusion and give us the opportunity to study organic basis of psychiatric disorders. Chikungunya infection is usually self-limiting and resolution occurs in a week [9]. In

Table 1. Summary findings.

\begin{tabular}{cc}
\hline Psychiatric symptoms & N (\%) \\
\hline Delirium (non-specified) & $45(77.5 \%)$ \\
Panic Disorder & $33(56.8 \%)$ \\
Phobic Disorder & $21(36.0 \%)$ \\
Neurasthenia or Fatigue Syndrome & $16(27.5 \%)$ \\
Hypersomnia & $8(13.7 \%)$ \\
Claustrophobia & $3(5.0 \%)$ \\
\hline
\end{tabular}


our study, 77\% children had Delirium(non-specified), it was significantly high in number. As per the parents, Delirium occur on second or third day of onset of the fever, it subsided after 48 to 72 hours. In a recently published paper reported about the psychiatric morbidities in adult patients with Chikungunya infection in India shown that depressive symptoms were found in 5, and anxiety symptoms in 3 patients among 20 patients with psychiatry complaints [8]. Our study revealed the difference in presentation of psychiatric complaints among the pediatric patients where the Delirium is more frequent than anxiety and depression, which are more frequent in adult patients. Moreover, according to our study, Panic Disorder, Phobic Disorder, Fatigue syndrome, Hypersomnia, and Claustrophobia are also important psychiatric complications in Chikungunya fever among children in Bangladesh. There is not much information about the pathogenesis of the central nervous system injury in such infections [10]. We assume that, children suffer from Chikungunya infection having high grade fever, which may cause delirium itself. Another explanation is that; the virus may change the neurochemical equilibrium in the brain which may produce the psychiatric symptoms. Moreover, the disease plays a role to produce psychological stress or mental trauma [5]. Study may need to know details pathophysiology of development of psychiatric symptoms of Chikungunya fever effected children.

\section{Conclusion}

This study suggested that psychiatric morbidity among pediatric Chikungunya patients is very common. Knowledge of the pathogenesis is required to reduce the psychiatric complications and prevent the injuries and morbidity. There is an emergence to invent the prophylactic vaccination, proper and early diagnosis of Chikungunya fever. Further research should be carried out regarding pathogenesis of the Chikungunya fever.

\section{References}

[1] Enserink, M. (2006) Infectious Diseases. Massive Outbreak Draws Fresh Attention to Little-Known Virus. Science, 311, 1085. https://doi.org/10.1126/science.311.5764.1085a

[2] Ravi, V. (2006) Re-Emergence of Chikungunya Virus in India. Indian Journal of Medical Microbiology, 24, 83-84. https://doi.org/10.4103/0255-0857.25175

[3] Khatun, S., Chakraborty, A., Rahman, M., NasreenBanu, N., Rahman, M.M., Hasan, S.M., Luby, S.P. and Gurley, E.S. (2015) An Outbreak of Chikungunya in Rural Bangladesh, 2011. PLOS Neglected Tropical Diseases, 9, e0003907. https://doi.org/10.1371/journal.pntd.0003907

[4] Martins, H.A.L., Bernardino, S.N., Ribas, K.H., Santos, C.C., Antunes, T., et al. (2016) Outbreak of Neuro-Chikungunya in Northeastern Brazil. Journal of Neuroinfectious Diseases, 7, 218. https://doi.org/10.4172/2314-7326.1000218

[5] Bhatia, M.S., Gautam, P. and Jhanjee, A. (2015) Psychiatric Morbidity in Patients with Chikungunya Fever: First Report from India. Journal of Clinical and Diagnostic Research, 9, VC01-VC03. https://doi.org/10.7860/JCDR/2015/14569.6586

[6] Ramchurn, S.K., Moheeput, K. and Goorah, S.S. (2009) An Analysis of a Short-Lived 
Outbreak of Dengue Fever in Mauritius. Euro Surveillance, 14, 19314.

https://doi.org/10.2807/ese.14.34.19314-en

[7] Rampal, S.M. and Meena, H. (2007) Neurological Complications in Chikungunya Fever. Journal of the Association of Physicians of India, 55, 765-769.

[8] Pialoux, G., Gaüzère, B.A., Jauréguiberry, S. and Strobel, M. (2007) Chikungunya, an Epidemic Arbovirosis. The Lancet Infectious Diseases, 7, 319-327. https://doi.org/10.1016/S1473-3099(07)70107-X

[9] Chhabra, M., Mittal, V., Bhattacharya, D., Rana, U. and Lal, S. (2008) Chikungunya Fever: A Re-Emerging Viral Infection. Indian Journal of Medical Microbiology, 26, 5-12. https://doi.org/10.4103/0255-0857.38850

[10] John, C.C., Carabin, H., Montano, S.M., Bangirana, P., Zunt, J.R. and Peterson, P.K. (2015) Global Research Priorities for Infections That Affect the Nervous System. Nature, 527, S178-S186. https://doi.org/10.1038/nature16033 